\title{
Molecular dynamics simulations of nanoscratching of 3C SiC
}

\author{
A. Noreyan ${ }^{\mathrm{a}}$, J.G. Amar ${ }^{\mathrm{b}, *}$ \\ a Department of Mechanical, Automotive, and Materials Engineering, University of Windsor, \\ Windsor, ON N9B3P4, Canada \\ b Department of Physics E' Astronomy, University of Toledo, \\ Toledo, $\mathrm{OH} 43606$, USA
}

\section{A R T I C L E I N F O}

\section{Article history:}

Received 5 April 2006

Received in revised form 16 January 2008

Accepted 18 February 2008

Available online 11 April 2008

\section{PACS:}

$62.25+\mathrm{g}$

62.20. $-\mathrm{X}$

60.20.Fe

Keywords:

Silicon carbide

Nanoscratching

Atomic scale friction

Amorphization

Molecular dynamics

\begin{abstract}
A B S T R A C T
We have carried out molecular dynamics simulations of nanoindentation followed by scratching at constant depth on the Si-terminated $(001)$ surface of $3 \mathrm{CSiC}$. The dependence of the friction coefficient, scratch hardness, and wear on scratching depth, velocity, direction, and indenter size and shape are investigated. In general, both the scratch hardness and friction coefficient increase with indentation depth but decrease with increasing scratching speed. We also find that the scratch hardness and friction coefficient are

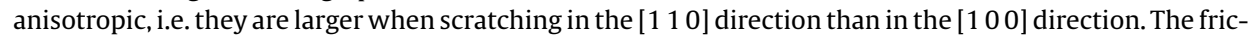
tion coefficient also depends on the rake angle of the tool and is larger for a pyramidal indenter, due to the presence of a large negative rake angle in this case. While the primary mode of wear observed for scratching is ploughing in good agreement with experimental studies, there is significantly more pile-up and chip formation in the [1 10 ] direction. We have also used the shortest path ring distribution, pair-correlation function, bond-angle distribution and bond number analysis to investigate the nature of the plastic deformation due to nanoscratching. We find that scratching leads to a partial amorphization of the material along the scratching trajectory. The size of the amorphization region increases with scratching velocity, thus explaining the decrease in scratch hardness and friction coefficient for higher scratching velocities.
\end{abstract}

(c) 2008 Elsevier B.V. All rights reserved.

\section{Introduction}

Due to its mechanical, thermal, and electrical properties, SiC is an important material for a variety of technological applications, ranging from high-temperature electronics to structural and protective components in nuclear fusion reactors [1]. In particular, because of its mechanical and thermal properties it plays an important role in a variety of mechanical devices including seal rings, journal bearings, valves, nozzles, rotors, as well as micro-mechanical devices. Consequently a variety of theoretical and experimental studies have been carried out to investigate the different properties and possible applications of this material [1-19].

Different polytypes of tetrahedrally bonded SiC exist at ambient pressure, which are differentiated by the stacking sequence of the tetrahedrally bonded $\mathrm{Si}-\mathrm{C}$ bilayers [1]. Among these polytypes, the $\beta$-SiC or $3 C$ (cubic) polytype, which has the zinc-blende structure, is of much interest. As a result, there have been a variety of experimental studies of the tribological properties of $\mathrm{SiC}$ [20-27]. However, despite these experimental studies, the fun-

\footnotetext{
* Corresponding author.

E-mail address: jamar@physics.utoledo.edu (J.G. Amar).
}

damental mechanisms of mechanical deformation are not well understood.

In order to try to understand these mechanisms, recently we have carried out a molecular dynamics (MD) study of the nanoindentation of $3 \mathrm{C} \mathrm{SiC}[28,29]$. Over the range of indenter sizes used in our simulations, we found that both the critical pressure and indentation depth for the elastic-to-plastic transition decreased somewhat with increasing indenter size. In contrast, the critical indentation depth for the elastic-to-plastic transition was not found to depend on the indenter velocity. Our results also indicated the existence of a phase transition from the cubic zinc-blende structure to the rocksalt structure at a critical indentation depth. In particular, for indentation depths beyond the critical depth, the pressure increases and saturates at $100 \mathrm{GPa}$, which corresponds to the experimental pressure at which $\beta$-SiC transforms to the rocksalt structure. This is in reasonable agreement with experimental studies of pressure-induced structural transformation in bulk SiC.

Here we present the results of molecular dynamics simulations of nanoindentation followed by nanoscratching of the silicon terminated $(001)$ surface of $3 \mathrm{C}$ silicon carbide by a diamond tip. These simulations were carried out to investigate the dependence of friction coefficient, friction force, abrasive wear, and scratch hardness on indentation depth, shape, scratching velocity and scratching direction. 


\section{Molecular dynamics simulations}

In order to study the nanoscratching of $\mathrm{SiC}$ by a diamond tip we have carried out molecular dynamics simulations using the Tersoff $\mathrm{SiC}$ potential [4]. We note that this potential, which includes a pairinteraction as well as a three-body interaction which depends on bond angles, has been shown to reproduce many of the mechanical and thermal properties of $3 \mathrm{C} \mathrm{SiC}$ as well as of several other $\mathrm{SiC}$ polytypes [4]. The Tersoff potential has also been shown [28] to reproduce the known $p(2 \times 1)$ reconstruction of the $3 C \operatorname{SiC}(001)$ surface in the absence of stress, in good agreement with density functional theory predictions [17].

In our simulations the workpiece was taken to have the $3 \mathrm{C}$ $\mathrm{SiC}$ cubic crystal structure (zinc-blende structure) with a lattice constant of $4.32 \AA$ corresponding to the room-temperature lattice constant using the Tersoff potential [4]. The single crystal workpiece was made from a stacking of $18 \mathrm{SiC}(001)$ bilayers with a free surface corresponding to the Si-terminated (001) surface. In order to hold the workpiece in place, fixed boundary conditions were assumed in the $x$ and $y$ directions (e.g. parallel to the surface) as well as on the bottom of the workpiece while the free surface was allowed to relax in the $z$ direction. Runs with periodic boundary conditions in the $x$ and $y$ directions were also carried out for comparison, and no difference was found between these results and those obtained with fixed boundary conditions. The workpiece was first equilibrated for a period of $14 \mathrm{ps}$, which was significantly longer than the time needed for the system to reach equilibrium. For simplicity, in all our simulations the diamond indenter was assumed to be rigid with a lattice constant of $3.57 \AA$. We note that this approximation has been used in a number of other studies [35-37] and may be justified by the fact that the hardness of diamond (10 on the Mohs scale) is significantly larger than that of SiC (8 on the Mohs scale). Both pyramidal and "rectangular" indenters were used with square tips and different tip areas as shown in Table 1 . In addition, two different workpiece sizes were used-one with 92,120 atoms $(207.4 \AA \times 103.7 \AA \times 43.2 \AA$ ) for scratching in the $[100]$ direction, and the other with 92,480 atoms $(146.9 \AA \times 146.9 \AA \times 43.2 \AA)$ for scratching in the [110] direction. We note that these sizes were selected to minimize boundary effects in the $y$ direction since the width of the workpiece was at least 5 times the width of the indenter as well as in the scratching $(x)$ direction since at the end of the scratch the distance between the indenter and the boundary (approximately $80 \AA$ ) was also significantly larger than the width of the indenter. In all cases, before indenting the indenter was initially held at a distance larger than the cutoff distance for the Tersoff SiC potential $\left(r_{\text {cut }}=3.0 \AA\right.$ ) from the surface of the workpiece.

While the main focus of this study is scratching, in order to determine the effects of the initial indentation speed on the results, we have carried out two types of indentation. In the first type, the indenter was moved towards the surface at a speed of $87.5 \mathrm{~m} / \mathrm{s}$ until the desired depth was reached and then the system was equilibrated. In the second type each $1.5 \AA$ Aepth increment was followed by a holding phase of about $5 \mathrm{ps}$ to allow the system to relax in order to simulate quasi-static indentation [35,37]. However, the scratching results were essentially the same in both cases. This

Table 1

Parameters describing diamond indenter used in scratching simulations

\begin{tabular}{llll}
\hline \# of atoms in indenter & Shape of indenter & Tip area $\left(\AA^{2}\right)$ & Tip width $(\AA)$ \\
\hline 1968 & Pyramidal & 205.6 & 14.3 \\
3368 & Pyramidal & 458 & 21.4 \\
1620 & Rectangular & 205.6 & 14.3 \\
2704 & Rectangular & 458 & 21.4 \\
\hline
\end{tabular}
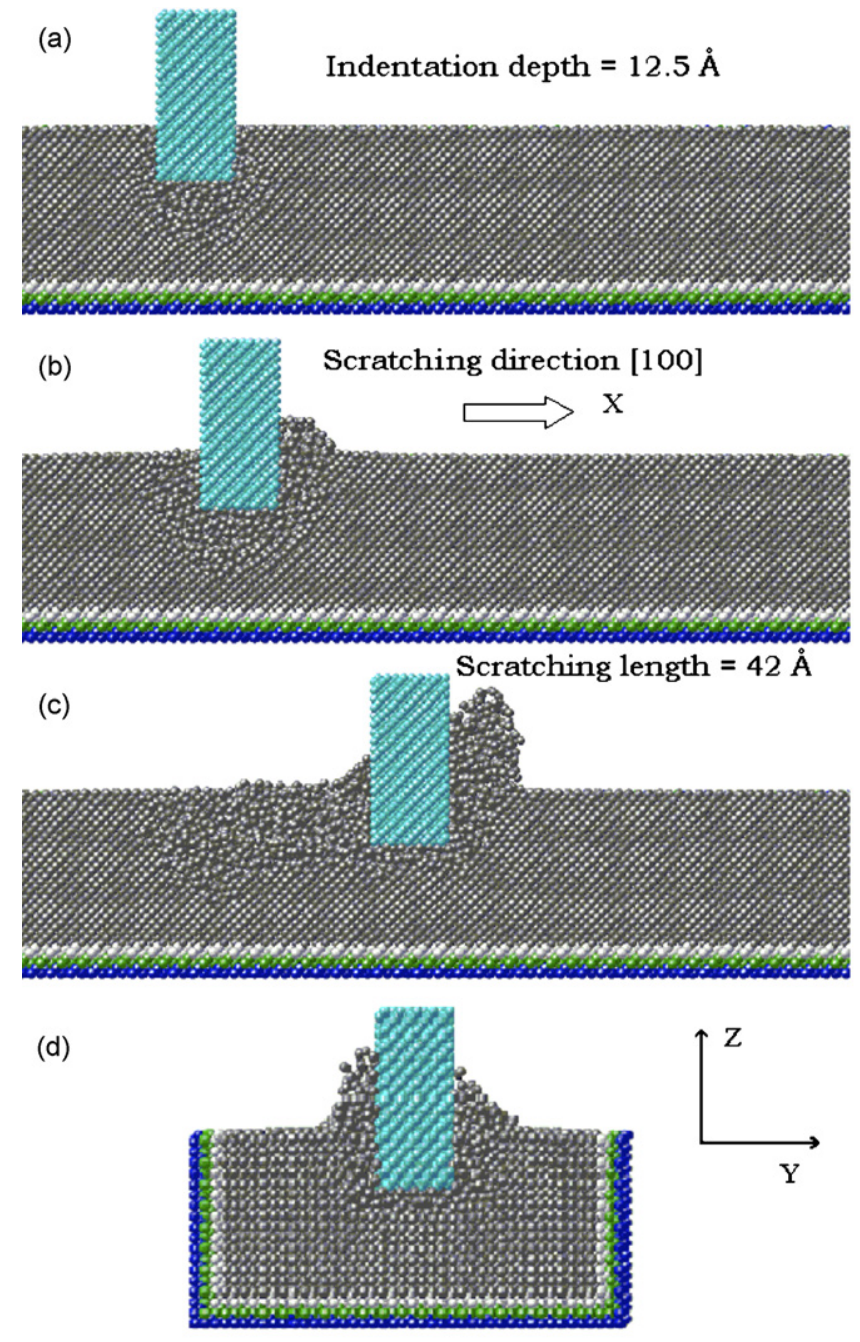

Fig. 1. Snapshots showing cross-sectional views for case of "rectangular" indenter of different stages of MD simulation of indentation to a depth of $12.5 \AA$ followed by scratching in the [1 00 ] direction at a speed of $214 \mathrm{~m} / \mathrm{s}$.

is consistent with our previous work [28] in which the indentation of $\mathrm{SiC}$ was studied and there was a negligible dependence on indentation velocity.

Fig. 1 shows the model used in our MD simulation studies. The workpiece is divided into three different zones: a "moving zone (gray atoms), a thermostat zone (white, green and blue atoms), and a fixed boundary zone (black atoms). The thermostat zone allows for a gradual transition from the pure MD region to the fixed region and also serves to equilibrate the system to the desired temperature as well as to eliminate reflections from the boundaries. Thus, while atoms in the "moving" zone undergo ordinary molecular dynamics, the motion of atoms in the thermostat zone is modified by the presence of a velocity-reset function [30-32]. In particular, after every timestep the velocities of the atoms in the inner layer of the thermostat zone (white atoms) are rescaled to the desired temperature, while the velocities of the atoms in the outer two layers of the thermostat zone (green and blue atoms) are re-assigned with a new thermal distribution corresponding to the desired temperature. The boundary atoms are fixed in position and serve to reduce the edge effects and maintain the proper symmetry of the crystal. All of our simulations were carried out at room temperature.

In order to study the dependence of the friction coefficient, wear, and scratch hardness on scratching velocity and scratching direc- 
tion, simulations were carried out with three different scratching velocities $(87.5,214$, and $428 \mathrm{~m} / \mathrm{s})$ and along the [ 100 ] and [ 1110$]$ directions. To evaluate the dependence of atomic scale friction on indentation depth, we considered four different indentation depths (3.5, 6.5, 9.5, and $12.5 \AA$ ). We note that in our simulations, the zero of indenter displacement was defined as the point at which the force between the workpiece and the indenter becomes repulsive as the tip approaches the surface. Constant indentation depth and scratching velocity were maintained during the entire scratching length. The total force on the substrate was calculated during indentation and scratching by measuring the change in the total force acting on the indenter atoms. The scratch hardness was then calculated as equal to the ratio of the (average) magnitude of the force applied in the scratching direction to the projected contact area, while the friction coefficient was calculated as the ratio of the magnitude of the component of the force in the scratching direction to the $z$-component of force. The projected contact area was assumed to correspond to the portion of the indenter beneath the substrate and did not include the area of the chip formed in front of the indenter. In order to speed up our molecular dynamics simulations, a parallel code based on atom-decomposition [33] was used, while the equations of motion were integrated using the velocity-Verlet algorithm with a timestep of $0.175 \mathrm{fs}$. Our simulations were carried out on the Ohio Supercomputer Center Pentium 4 Cluster. On 26 processors a system of 93,132 atoms runs at a rate of about $2.2 \mu \mathrm{s} / \mathrm{atom} /$ timestep.

\section{Results}

Fig. 1 shows cross-sectional side views (Fig. 1(a)-(c)) and a cross-sectional back view (Fig. 1(d)) obtained from simulations of scratching in the [1 000 ] direction after indentation to a depth of $12.5 \AA$ for the case of a small rectangular indenter (tip width $14.3 \AA$ ). As indicated by our previous nanoindentation simulations [28], the indentation depth used in all our scratching simulations is significantly beyond that corresponding to the critical distance (approximately $2 \AA$ [28]) for plastic deformation [28]. As a result, by the end of the indentation process (Fig. 1(a)) the region directly beneath the indenter has undergone a structural transformation from the four coordinated zinc-blende structure to the six coordinated rocksalt structure [28].

Fig. 1(b) shows the deformation below, behind, and in front of the indenter shortly after scratching has begun and the indenter has moved a distance of $7 \AA$, while the final stage of the scratching process is shown in Fig. 1(c) and (d). As can be seen there is initially a considerable amount of subsurface deformation after indentation, which extends several layers below the indentationscratching depth. However, the deformation zone is localized near the indenter and, as discussed in more detail below, there is no evidence of dislocations. In contrast, after scratching there is a considerable amount of material removed from the substrate both in front of, as well as on the sides of the indenter. The material removal mechanism is predominantly ploughing (see Fig. 1(d)). Due to this removal, the size of the deformed region directly underneath the indenter during and after scratching is significantly smaller than after the initial indentation.

Fig. 2 shows typical results for the forces on the indenter in the scratching $(x)$ direction as well as normal to the substrate $(z$ direction) as a function of displacement. Here the zero of displacement along the $x$-axis corresponds to the onset of scratching after indenting $12.5 \AA$ and equilibrating the system for 5.0 ps. During indentation (not shown) the normal force increases rapidly while the average force parallel to the surface remains almost zero. However, during the scratching process, the normal force initially drops significantly, while the (negative) friction force increases in magni-

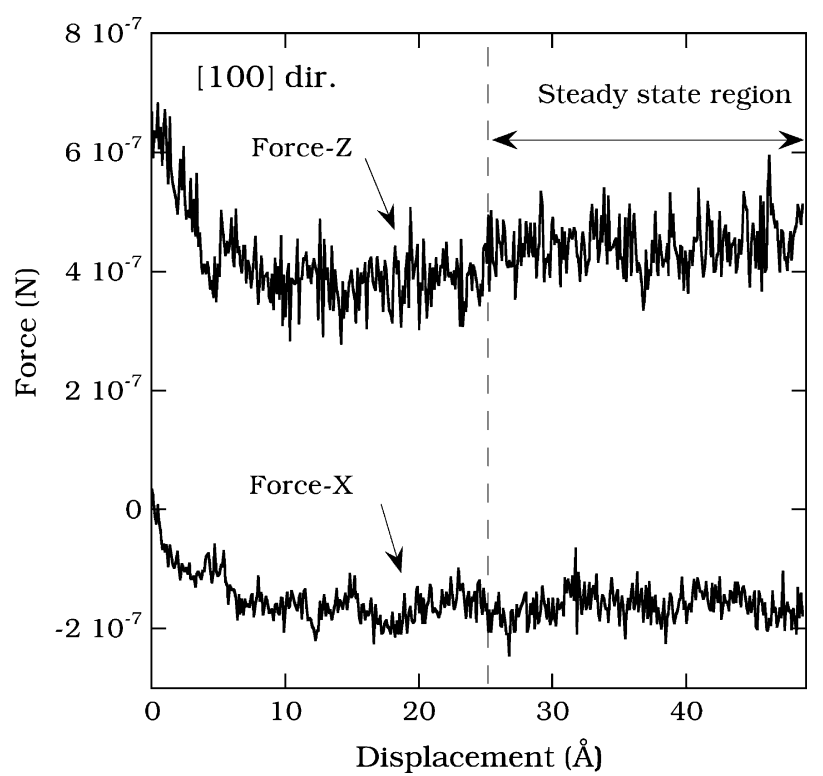

Fig. 2. Force in $x$ and $z$ directions as function of $x$-displacement for scratching in

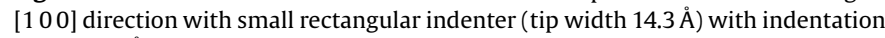
depth $12.5 \AA$, and scratching speed of $214 \mathrm{~m} / \mathrm{s}$.

tude. After a displacement of approximately $28 \AA$, a steady state is reached such that both the normal and tangential forces are essentially constant, fluctuating around an average value. Using the data in this steady-state region we obtain a friction coefficient $f=0.37 \pm 0.02$ and a scratch hardness $H=89 \mathrm{GPa}$.

Figs. 3 and 4 show similar results for the case of scratching in the [ 1110 ] direction. In this case the scratching speed was $214 \mathrm{~m} / \mathrm{s}$ and again the small rectangular indenter (tip width $w=14.3 \AA$ ) was used with a scratching depth of $12.5 \AA$. For this scratching geometry a significantly larger scratching distance is needed to reach the steady state. As can be seen, both the average normal force and tangential force are larger than for scratching in the [ 100$]$ direction. Accordingly the friction coefficient and scratch hardness ( $f=1.21 \pm 0.02$ and $H=332 \mathrm{GPa}$ ) are significantly larger than for scratching in the [100] direction. This is consistent with the fact that the distance between [1 10 ] planes is significantly smaller than the distance between [ 100 ] planes, thus leading to increased

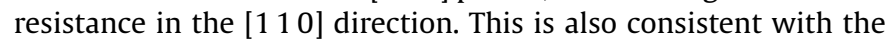
observation (see Figs. 1 and 3 ) that there is significantly more chip formation as well as ploughing for the case of scratching in the [1 110$]$ direction than in the [1 00 ] direction.

A summary of our results for the dependence of the friction coefficient and scratch hardness on indentation depth, scratching velocity and scratching direction for a small rectangular indenter $(T=300 \mathrm{~K})$ is shown in Tables 2 and 3 . In general, the friction coefficient and scratch hardness decrease as the scratching speed is increased, although the change is smaller for larger indentation

Table 2

Dependence of friction coefficient for small rectangular indenter on indentation depth, scratching velocity and scratching direction

\begin{tabular}{llll}
\hline Indenter depth $(\AA)$-direction & $87.5 \mathrm{~m} / \mathrm{s}$ & $214 \mathrm{~m} / \mathrm{s}$ & $428 \mathrm{~m} / \mathrm{s}$ \\
\hline $3.5-\left[\begin{array}{lll}1 & 0 & 0\end{array}\right]$ & $0.25 \pm 0.02$ & - & $0.24 \pm 0.02$ \\
$6.5-\left[\begin{array}{lll}1 & 0 & 0\end{array}\right]$ & $0.42 \pm 0.06$ & $0.29 \pm 0.03$ & $0.26 \pm 0.01$ \\
$9.5-\left[\begin{array}{lll}1 & 0 & 0\end{array}\right]$ & $0.53 \pm 0.02$ & $0.35 \pm 0.02$ & $0.28 \pm 0.02$ \\
$12.5-\left[\begin{array}{lll}1 & 0 & 0\end{array}\right]$ & - & $0.37 \pm 0.02$ & - \\
$3.5-\left[\begin{array}{lll}1 & 1 & 0\end{array}\right]$ & - & $1.1 \pm 0.03$ & - \\
$9.5-\left[\begin{array}{lll}1 & 1 & 0\end{array}\right]$ & - & $1.14 \pm 0.03$ & - \\
$12.5-\left[\begin{array}{lll}1 & 1 & 0\end{array}\right]$ & - & $1.21 \pm 0.02$ & - \\
\hline
\end{tabular}



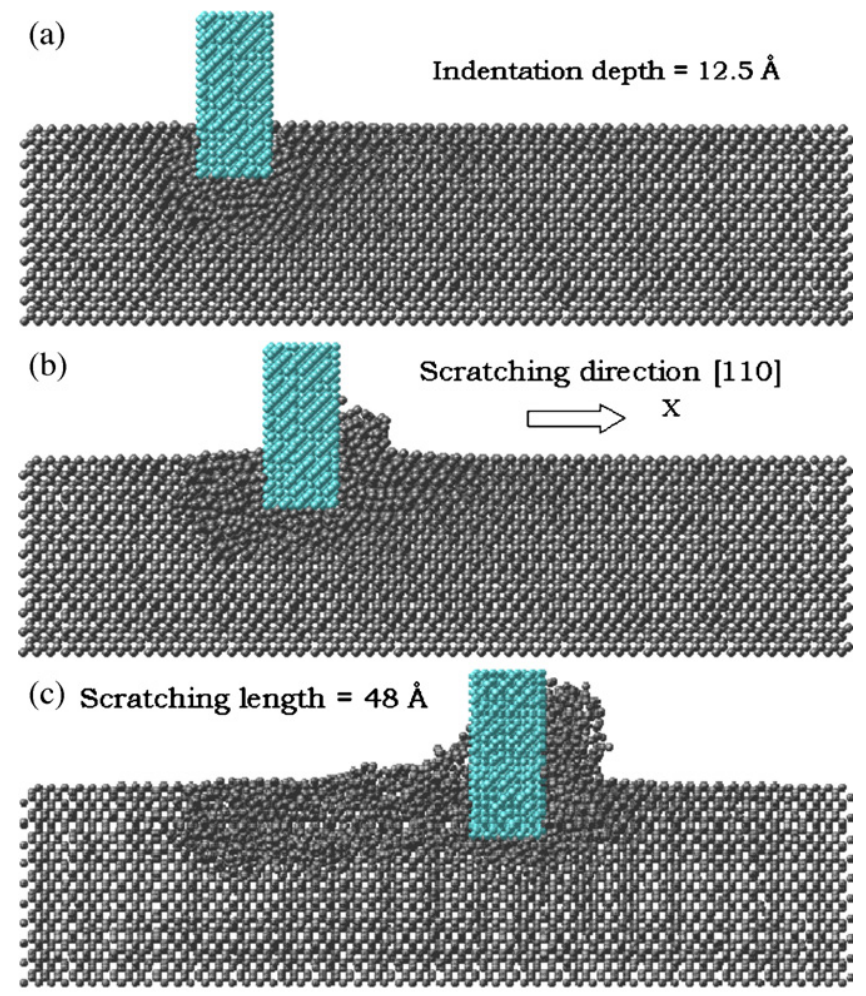

(d)

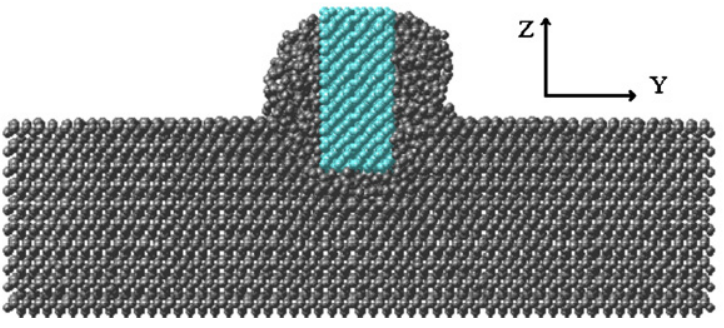

Fig. 3. Snapshots showing cross-sectional views of different stages of MD simulation of indentation $(12.5 \AA)$ followed by scratching in [1 10 ] direction with speed of $214 \mathrm{~m} / \mathrm{s}$.

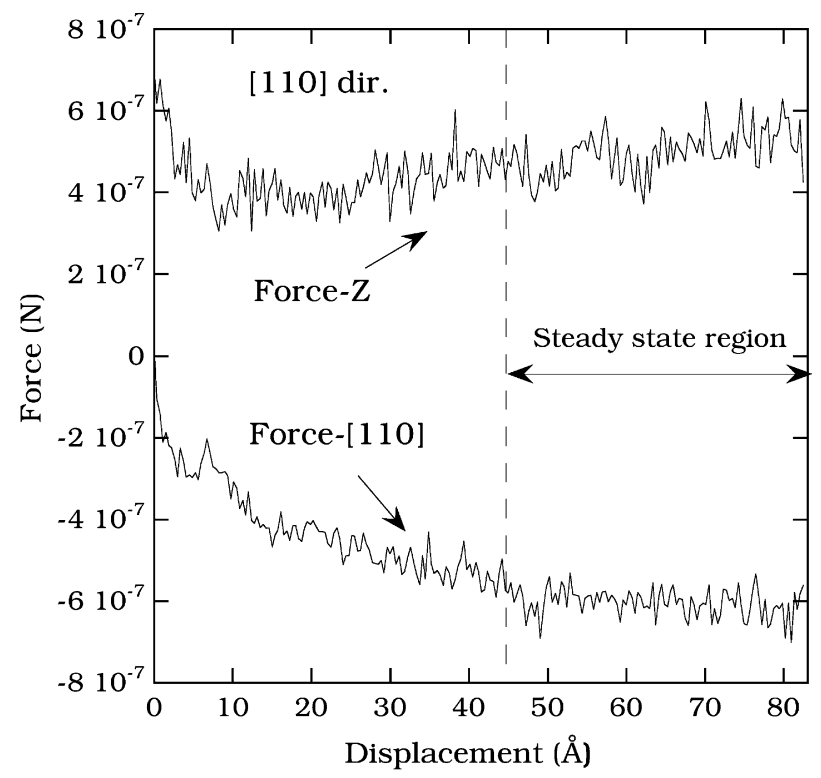

Fig. 4. Force along scratch direction ("Force-[1 10 ]") and perpendicular to substrate ("Force-Z") as function of displacement for scratching in [1 110$]$ direction with small rectangular indenter (tip width $14.3 \AA$ ) with indentation depth $12.5 \AA$, and scratching speed of $214 \mathrm{~m} / \mathrm{s}$.
Table 3

Dependence of scratch hardness (GPa) for small rectangular indenter on indentation depth, scratching velocity and scratching direction

\begin{tabular}{lccc}
\hline Indenter depth $(\AA)$-direction & $87.5 \mathrm{~m} / \mathrm{s}$ & $214 \mathrm{~m} / \mathrm{s}$ & $428 \mathrm{~m} / \mathrm{s}$ \\
\hline $3.5-\left[\begin{array}{lll}1 & 0 & 0\end{array}\right]$ & 69 & - & 72 \\
$6.5-\left[\begin{array}{lll}1 & 0 & 0\end{array}\right]$ & 95 & 70 & 63 \\
$9.5-\left[\begin{array}{lll}1 & 0 & 0\end{array}\right]$ & 115 & 76 & 73 \\
$12.5-\left[\begin{array}{lll}1 & 0 & 0\end{array}\right]$ & - & 89 & - \\
$3.5-\left[\begin{array}{lll}1 & 1 & 0\end{array}\right]$ & - & 284 & - \\
$9.5-\left[\begin{array}{lll}1 & 1 & 0\end{array}\right]$ & - & 319 & - \\
$12.5-\left[\begin{array}{lll}1 & 1 & 0\end{array}\right]$ & - & 332 & - \\
\hline
\end{tabular}

depths. The scratching speed dependence may be explained by the fact that the steady-state temperature in the scratching region increases as the scratching speed is increased. (For the case of a small indenter and indentation depth of $9.5 \AA$, and scratching in the [ 100 ] direction, the steady-state temperature increases from $342 \mathrm{~K}$ for $v=87.5 \mathrm{~m} / \mathrm{s}$ to $452 \mathrm{~K}$ for $v=428 \mathrm{~m} / \mathrm{s}$.) The increase in temperature leads to a "softening" of the material, producing a smaller friction force and scratch hardness. We note that a similar tendency has also been observed in experiments [26].

Our results also indicate that the friction coefficient and scratch hardness increase with indentation depth. As already noted, in the case of scratching in the $\left[\begin{array}{lll}1 & 1 & 0\end{array}\right]$ direction, the friction coefficient and scratch hardness are significantly larger than in the [1 00 0] direction. However, the dependence of the friction coefficient on the indentation depth is smaller in the [110] direction than in the [100] direction.

In order to investigate the dependence on indenter size, we have also carried out simulations of scratching in the [1 000$]$ direction with a large rectangular indenter (21.4 $\AA$ width) with a scratching speed of $214 \mathrm{~m} / \mathrm{s}$. As shown in Table 4, we find that both the friction coefficient and hardness are somewhat larger for the large rectangular indenter than for the small rectangular indenter. To investigate the dependence on indenter shape, we have also carried out simulations with pyramidal indenters corresponding to a negative rake angle of $45^{\circ}$. In this case we found that the material removal process was also due to ploughing, while the amount of deformation ahead of the tool as well as the degree of compression were larger (for the same size indenter) than for a rectangular indenter. We also found that the friction coefficient was somewhat larger than for the rectangular indenter. These results are qualitatively similar to those found in Refs. $[30,34]$ for the scratching of aluminum.

We now discuss the nature of the deformation due to scratching in more detail. In order to investigate the nature of the deformation we have carried out a bond-number analysis for the substrate atoms near the indenter. Fig. 5 presents side and front views of the final frame in the case of scratching in the [100] direction with two different scratching speeds for a scratching depth of $9.5 \AA$. Only atoms with other than four bonds are shown and the colors correspond to the number of bonds. The horizontal "line" of gray atoms corresponds to the surface layer of the workpiece with only two bonds. Here we have assumed that two atoms share a bond if their distance is less than $2.05 \AA$. As can be seen, for both scratching speeds the atoms with other than four bonds are localized around the indenter. In addition most of the non-four-bonded atoms below

Table 4

Dependence of friction coefficient for large rectangular indenter on indentation depth with $214 \mathrm{~m} / \mathrm{s}$ scratching velocity

\begin{tabular}{lll}
\hline Indenter depth $(\AA)$ & Friction coefficient & Scratch hardness $(\mathrm{GPa})$ \\
\hline $6.7-\left[\begin{array}{lll}1 & 0 & 0\end{array}\right]$ & $0.32 \pm 0.01$ & 89 \\
$9.7-\left[\begin{array}{lll}1 & 0 & 0\end{array}\right]$ & $0.39 \pm 0.01$ & 91 \\
\hline
\end{tabular}




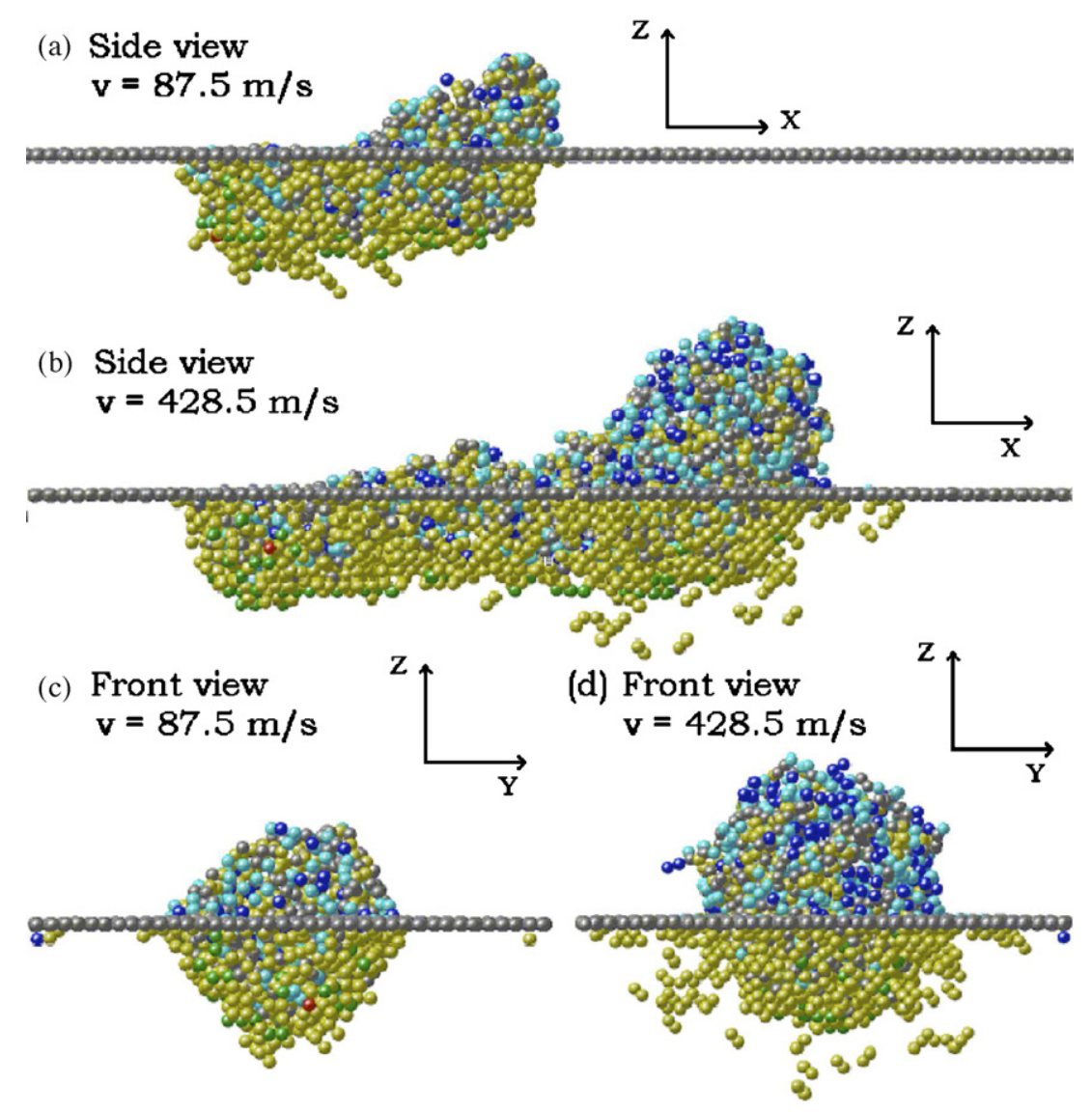

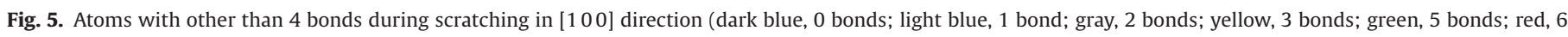

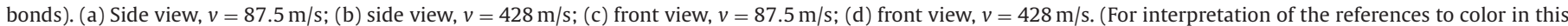
figure legend, the reader is referred to the web version of the article.)

the surface typically have only 3 bonds (yellow) although there are a few with 5 bonds (green). In contrast, the atoms above the surface include a significant fraction of both singly bonded (dark blue) and zero-bonded (light blue) atoms in addition to a large number of atoms with only 3 bonds (yellow). We note that for the higher speed scratch $(v=428 \mathrm{~m} / \mathrm{s})$, the deformation region is somewhat larger while the shape of the deformation region is less well defined than for the shorter speed scratch $(v=87.5 \mathrm{~m} / \mathrm{s})$.

In order to further understand the nature of the plastic deformation due to nanoscratching, we have also measured the pair-correlation function (p.c.f.) in a rectangular region of size $24 \AA \times 24 \AA \times 21 \AA$ surrounding the indenter as shown in Fig. 6. In agreement with our previous nanoindentation simulations [28], indentation to a depth of $9.5 \AA$ leads to an extra peak in the pair-correlation function at $2.5 \AA$ corresponding to the rocksalt structure. However, after scratching this peak disappears while the other two peaks at 1.87 and $3 \AA$ which are common to the $3 \mathrm{C}$ and rocksalt structures become significantly weaker. This indicates a transition back to a somewhat disordered structure which is similar to the original $3 \mathrm{C}$ structure.

As shown in Fig. 7, we have also measured the bond-angle distribution before indentation, after indentation, and after scratching in order to further understand the structural changes due to scratching. We note that as for the pair-correlation function, the peaks that were generated due to indentation become significantly weaker during scratching (Fig. 7). Instead the bond-angle distribution is closer to the initial zinc-blende structure, with some distortions, again indicating a transition to a somewhat disordered structure which is similar to the original $3 \mathrm{C}$ structure.
In order to further understand the subsurface deformation due to scratching, we have also measured the shortest path ring distribution [35,38-40]. The idea behind this method is that changes in the shortest path ring sizes indicate the presence of struc-

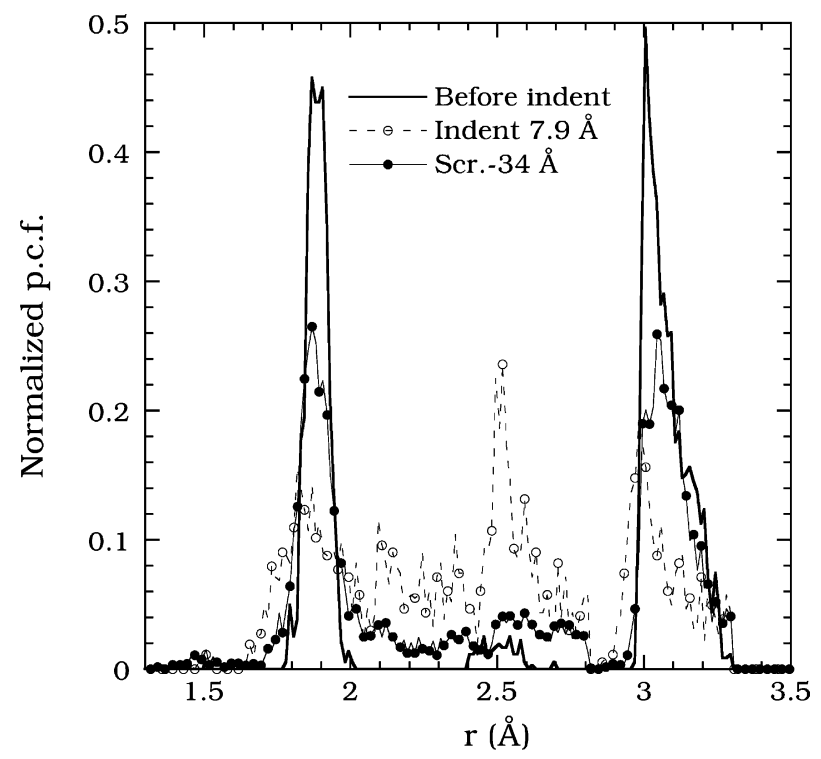

Fig. 6. Normalized pair-correlation function (p.c.f.) before indentation, after indentation, and after scratching for scratching with small indenter (indentation depth $9.5 \AA$, scratching speed $85.7 \mathrm{~m} / \mathrm{s}$, and scratching distance of $34.5 \AA$ ). 


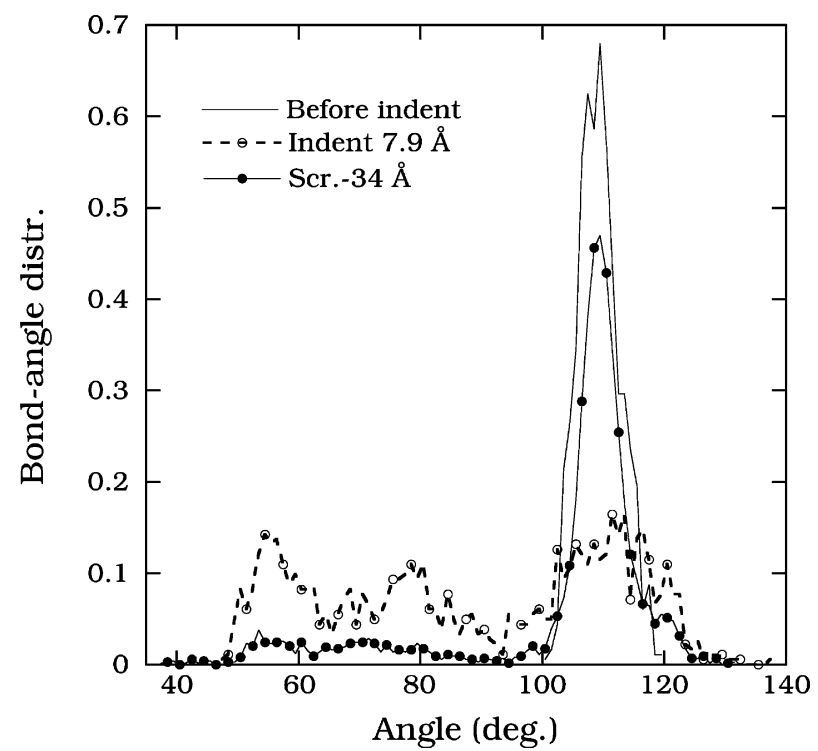

Fig. 7. Bond-angle distribution for scratching with small indenter before indentation (solid line), after indentation to depth of $9.5 \AA \AA$, and after scratching over a distance of $34.5 \AA$. Scratching speed is $85.7 \mathrm{~m} / \mathrm{s}$.

tural deformation. In particular, the shortest path ring distribution has been used to study the short and intermediate-range structural correlations in amorphous silicon carbide [40], as well as to investigate the nature of plastic deformation due to nanoindentation on (111) surface of silicon carbide [35], and to explain the relationship of chemical disorder to topological disorder in irradiation-amorphized silicon carbide [39]. It has also been used to visualize the presence of dislocations [35]. We note that the shortest path rings in the $3 \mathrm{C} \mathrm{SiC} \mathrm{structure} \mathrm{are} \mathrm{all} \mathrm{three-fold} \mathrm{(six} \mathrm{atom)} \mathrm{rings}$ corresponding to three pairs of $\mathrm{Si}-\mathrm{C}$ bonds. Thus, the occurrence of structural deformation is marked by the presence of non-three-fold rings.

In our simulations, the distribution of $n$-fold rings was determined as follows. First the list of all nearest neighbors (NN) was constructed for all $\mathrm{Si}$ and $\mathrm{C}$ atoms using a nearest neighbor distance cutoff of $2.2 \AA$ which is slightly larger than the equilibrium

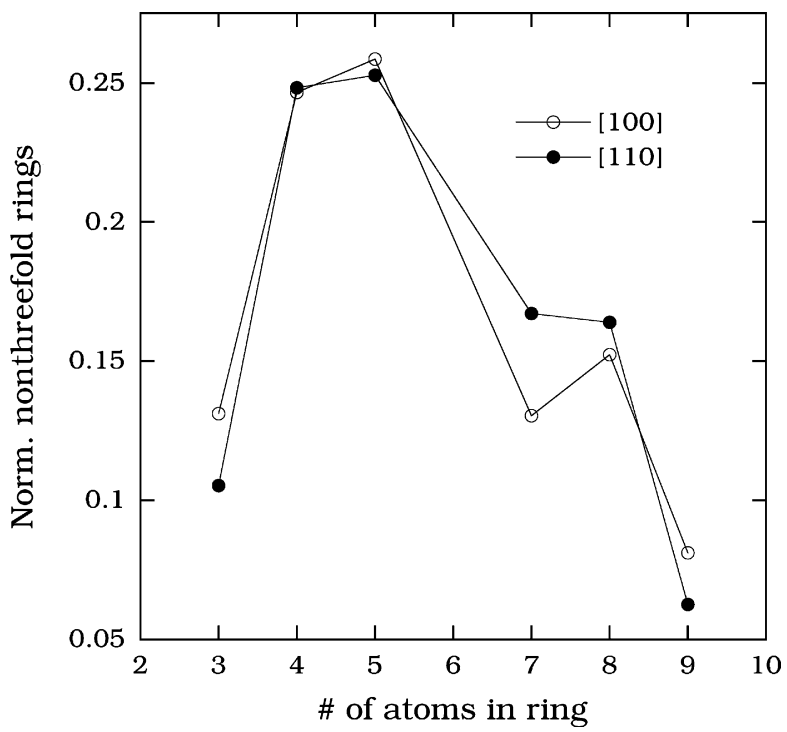

Fig. 8. Non-three-fold ring distribution $\left(n_{\text {ring }} \neq 6\right)$ for scratching in the $[100]$ and [1 110 0] directions with scratching speed $214 \mathrm{~m} / \mathrm{s}$ and scratching depth of $9.66 \AA$

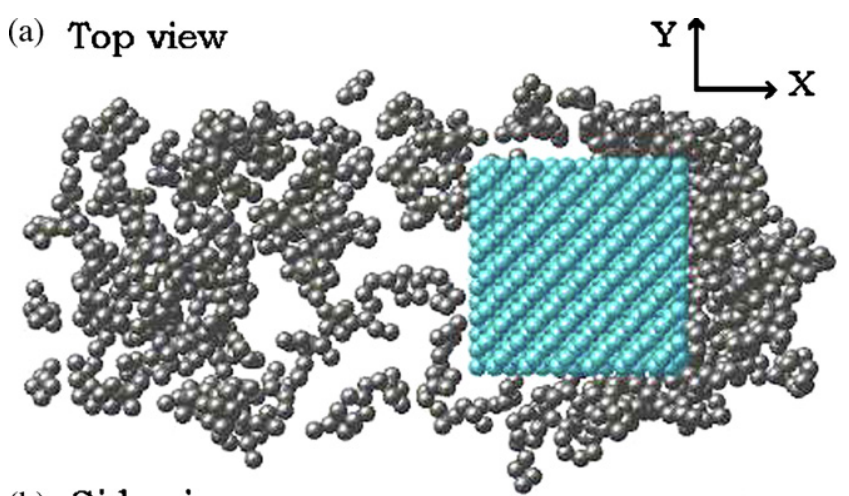

(b) Side view

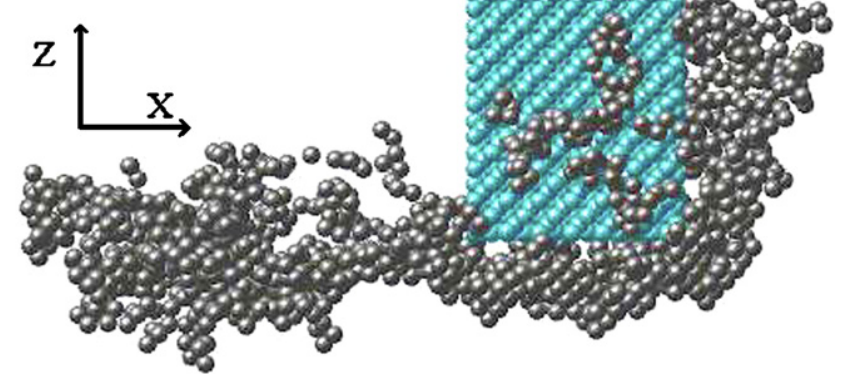

Fig. 9. Atoms which contain only non-three-fold rings for scratching in the [1 00$]$ direction and the same conditions as Fig. 8.

bond-length for $3 \mathrm{C} \mathrm{SiC}$. Then for each atom in the region surrounding the scratching trajectory, the shortest path ring corresponding to an alternating sequence of $\mathrm{Si}-\mathrm{C}$ and $\mathrm{C}-\mathrm{Si}$ bonds was determined. We define $n_{\text {ring }}$ as the number of atoms in the shortest path ring.

Fig. 8 shows our results for the ring-size distribution excluding three-fold rings $\left(n_{\text {ring }}=6\right)$ normalized over the total number of non-three-fold rings for the case of scratching in the [ 100$]$ and [1 110$]$ directions. We note that the ring-size distribution is different from that which occurs during indentation for which a peak at $n_{\text {ring }}=4$ corresponding to a phase transformation to the rocksalt structure was observed [28,29]. In particular, in both scratching directions we observe a peak corresponding to $n_{\text {ring }}=5$ which is consistent with a partial amorphization of the crystal. This is also consistent with the fact that as shown in Fig. 9 the deformation zone is localized near the indenter, in contrast to the case of scratching on aluminum $[30,34]$ in which extended slip planes propagating dislocations are observed. Thus, our ring-size distribution measurements indicate the existence of partial amorphization near the indenter during scratching rather than the formation of dislocations. Such a partial amorphization or disordering may also explain the fact that the scratch hardness is somewhat lower than the indentation hardness, since the hardness of amorphous silicon carbide is known to be smaller than that for crystalline structures [41].

\section{Conclusion}

We have carried out molecular dynamics simulations of nanoscratching on the Si terminated ( 001 ) surface of $3 \mathrm{C} \mathrm{SiC}$ using both rectangular and pyramidal indenters. In particular, the dependence of the friction coefficient, scratch hardness, and wear on scratching depth, scratching velocity, scratching direction, and indenter size have been studied. In general, we find that the friction coefficient and scratch hardness increase with indentation depth but decrease with increasing scratching velocity. However, at high scratching velocities the dependence on indentation depth is relatively weak. We have also found significant anisotropy in the hardness and fric- 
tion coefficient. In particular, both the hardness and friction coefficient are significantly larger in the [ 110$]$ direction than in the [ 100$]$ direction. We note that the direction dependence of the friction coefficient is weaker at high scratching velocity. This is consistent with the fact that more amorphization is observed at higher speeds.

By analyzing the pair-correlation function, bond-angle distribution, and ring-size distribution in the region surrounding the indenter, we have also studied the nature of the deformation during scratching. As indicated by the decreased peak heights observed in the pair-correlation function and bond-angle distributions, and further supported by our results for the ring-size distribution, one major effect of scratching on the substrate - in addition to "ploughing" - is the partial amorphization of the material along the scratching trajectory. This is also consistent with the fact that we could not observe any dislocations or dislocation loops generated due to nanoscratching. We note that the size of the region of amorphization increases with increasing scratching velocity. This increase in the size of the amorphization region may also explain the decrease in scratch hardness and friction coefficient for higher scratching velocities. These results may also explain recent experimental results on grinding of polycrystalline $\mathrm{SiC}$, in which the possibility of grinding in ductile mode at high speed has been observed [42].

Finally, it is interesting to compare our results for the friction coefficient with experimental results obtained for significantly larger indenter sizes $(12.7 \mathrm{~mm})$ and significantly lower scratching speeds $(0.0014-0.42 \mathrm{~m} / \mathrm{s})$. Dong et al. [27] reported an average friction coefficient $f=0.23$ for polycrystalline $3 \mathrm{C} \mathrm{SiC}$ at room temperature, while Li et al. [24] found that the friction coefficient of $\mathrm{SiC}$ is higher than 0.3 under dry friction [24]. Our simulation results for the friction coefficient in the [1 00 ] direction (ranging from 0.24 to 0.53 depending on the indentation depth and velocity) are in reasonable agreement with these values although they tend to be somewhat higher. In contrast, our results for the friction coefficient in the [110] direction $(f=1.1-1.2)$ are significantly higher. However, this may not be surprising since the existence of polycrystallinity as well as defects and/or an oxidation layer in experiments tends to decrease the friction coefficient [21]. We thus conclude that our results are in reasonable qualitative agreement with the existing experimental results for larger indenter sizes.

\section{Acknowledgments}

This research was supported by the NSF through Grants No. CCF0428826 and DMR-0606307. We would also like to thank the Ohio Supercomputer Center for a grant of computer time.

\section{References}

[1] H. Markoc, S. Strite, G. Gao, M. Lin, B. Sverdlov, M. Burns, J. Appl. Phys. 76 (1994) 1363.

[2] M. Yoshida, A. Onodera, M. Ueno, K. Takemura, O. Shimomura, Phys. Rev. B 48 (1993) 10587.

[3] F. Shimojo, I. Ebbsjo, R.K. Kalia, A. Nakano, J.P. Rino, P. Vashishta, Phys. Rev. Lett. 84 (2000) 3338.

[4] J. Tersoff, Phys. Rev. B 39 (1989) 5566.

[5] C. Kohler, Phys. Status Solidi (b) 234 (2002) 522-540.

[6] L. Malerba, J.M. Perlado, Phys. Rev. B 65 (2002) 045202.

[7] R. Devanathan, W. Weber, F. Gao, J. Appl. Phys. 90 (2001) 2303.

[8] F. Gao, E. Bylaska, W. Weber, L. Corrales, Nucl. Instrum. Methods B 180 (2001) 286.

[9] M. Tang, S. Yip, Phys. Rev. B 52 (1995) 15150.

[10] L. Porter, J. Li, S. Yip, J. Nucl. Mater. 246 (1997) 53-59.

[11] H. Yan, A.P. Smith, H. Jonsson, Surf. Sci. 330 (1995) 265-275.

[12] K. Karch, P. Pavone, W. Windl, O. Schutt, D. Strauch, Phys. Rev. B 50 (1994) 17054.

[13] F. Gao, W.J. Weber, Nucl. Instrum. Methods B 191 (2002) 504-508.

[14] K. Karch, F. Bechstedt, P. Pavone, D. Strauch, Phys. Rev. B 53 (1996) 13400.

[15] K. Karch, P. Pavone, A.P. Mayer, F. Bechstedt, D. Strauch, Phys. B 219 \& 220 (1996) 448-450.

[16] G. Galli, L. Pizzagalli, A. Catellani, F. Gygi, A. Baratoff, Appl. Surf. Sci. 162-163 (2000) 1-8.

[17] A. Catellani, L. Pizzagalli, G. Galli, Mater. Sci. Eng. B 96 (2002) 132.

[18] M.S. Miao, M. Prikhodko, W.R.L. Lambrecht, Phys. Rev. B 66 (2002) 064107.

[19] M. Prikhodko, M.S. Miao, W.R.L. Lambrecht, Phys. Rev. B 66 (2002) 125201.

[20] K. Zum Gahr, R. Blattner, D. Hwang, K. Pohlmann, Wear 250 (2001) 299.

[21] K. Pohlmann, B. Bhushan, K. Zum Gahr, Wear 237 (2000) 116

[22] V. Murthy, H. Kobayashi, N. Tamari, S. Tsurekawa, T. Watanabe, K. Kato, Wear 257 (2004) 89.

[23] S. Sundararajan, B. Bhushan, Wear 217 (1998) 251.

[24] J. Li, J. Huang, S. Tan, Z. Cheng, C. Ding, Wear 218 (1998) 167.

[25] P. Vantomme, P. Deprez, A. Placet, D. Gaillot, Trib. Lett. 8 (2000) 51.

[26] H. Heshmat, S. Jahanmir, Trib. Lett. 17 (2004) 359.

[27] X. Dong, S. Jahanmir, L. Ives, Trib. Int. 28 (1995) 559.

[28] A. Noreyan, J. Amar, I. Marinescu, Mater. Sci. Eng. B 117 (2005) 235.

[29] A. Noreyan, Molecular dynamics simulations of nanoindentation and nanoscratching of SiC, Ph.D. Thesis, University of Toledo, 2005.

[30] R. Komanduri, N. Chandrasekaran, L. Raff, Wear 240 (2000) 113.

[31] R. Komanduri, N. Chandrasekaran, L. Raff, Philos. Mag. B 77 (1998) 7.

[32] M. Riley, M. Coltrin, J. Chem. Phys. 88 (1988) 5934.

[33] S. Plimpton, J. Comp. Phys. 117 (1995) 1.

[34] R. Komanduri, N. Chandrasekaran, L. Raff, Wear 242 (2000) 60.

[35] I. Szlufarska, R. Kalia, A. Nakano, P. Vashishta, Appl. Phys. Lett. 85 (2004) 378.

[36] Ph. Walsh, R. Kalia, A. Nakano, P. Vashishta, Appl. Phys. Lett. 77 (2000) 4332.

[37] I. Szlufarska, R. Kalia, A. Nakano, P. Vashishta, Phys. Rev. B 71 (2005) 174113.

[38] J. Rino, I. Ebbsjo, R. Kalia, A. Nakano, P. Vashishta, Phys. Rev. B 47 (1993) 3053.

[39] X. Yuan, L. Hobbs, Nucl. Instrum. Methods Phys. Res. B 191 (2002) 74.

[40] J. Rino, I. Ebbsjo, R. Kalia, A. Nakano, F. Shimojo, P. Vashishta, Phys. Rev. B 70 (2004) 045207.

[41] M. El Khakani, M. Chaker, A. Jean, S. Boily, J. Kieffer, M. O’Hern, M. Ravet, F. Rousseaux, J. Mater. Res. 9 (1994) 96.

[42] J. Patten, private communication. 